Recurrent respiratory infection may be a feature of patients who have deficiency of humoral or antibody response to some respiratory pathogens and to some antibody challenge tests whilst maintaining apparently normal levels of immunoglobulins (dysgammaglobulinaemia). Other patients may lack cellular immunity.

The conditions mentioned above are illustrated by reference to case histories.

\section{THE HISTOCHEMISTRY AND FINE STRUCTURE OF EPITHELIOID CELLS IN SARCOIDOSIS}

W. JONES WILLIAMS, D. ERASMUS, E. M. VALERIE JAMES and D. WILLIAMS Epithelioid cells in the granulomas of sarcoidosis, Kveim tests, tuberculosis, chronic beryllium disease, Crohn's disease and farmer's lung on light microscopy show similar histochemical features. They are rich in lysosomal enzymes and residual bodies (end products of lysosomal digestion) and show high pentose cycle, biosynthetic, enzyme activity. We considered that the biosynthetic activity is concerned with the production of lysosomal enzymes and that the cells were therefore primarily phagocytic.

On electron microscopy, epithelioid cells in sarcoidosis, Kveim tests and tuberculosis appear as two probably related cell types, $\mathbf{A}$ and $\mathbf{B}$. Both $\mathbf{A}$ and $\mathbf{B}$ cells show very scanty pinocytotic vesicles and occasional 'dense' (residual) bodies, which suggests that they are not primarily phagocytic. However, both types show morphological features suggesting active biosynthesis. A cells may be producing protein and B cells lipo- and mucoproteins.

We are currently attempting to characterize these cells further by electron microscope histochemistry.

\section{PULMONARY MECHANICS AND SURFACTANT FOLLOWING RE-IMPLANTATION OF DOG'S LUNG}

J. C. R. LINCOLN, N. BARNES, T. GOULD and E. O. REYNOLDS Eighteen dogs were studied before, during and immediately after autotransplantation of the left lung; 13 survivors were studied at intervals. Total pulmonary compliance, and that of the left lung after removal, pulmonary surfactant and lung stability were measured.

Pulmonary surfactant was normal in all cases studied from 3 days to 22 weeks. There was considerable reduction in total lung compliance with the chest closed after autotransplantation; this value returned to normal within 14 days. Composite pressure volume curves showed a reduction in volume of the autotransplanted lung at any time from 8 days to 22 weeks.

Pulmonary surfactant and lung stability remained unchanged in the autotransplanted lung when studied 3 days to 22 weeks after operation. There was an immediate fall in total lung compliance during the operative procedure which returned to normal within 14 days. Since there was a $33 \%$ reduction in volume in the autotransplanted lung from 1 to 22 weeks after the procedure, despite normal values for surfactant, it is suggested that early changes in compliance are due to acute interstitial oedema. The long-term reduction in volume with normal pulmonary surfactant is probably due to permanent interstitial changes.

\section{PATTERNS OF BREATHING IN HEALTH AND DISEASE}

A. R. TANSER An impedance pneumograph provides a method of recording breathing from electrodes on the chest wall. Under suitable conditions the record is quantitative, and recording may be continued for long periods without interference to the patient.

There is a wide variation in the pattern of breathing of normal subjects. Disease of the central nervous system, cardiovascular system or respiratory system may be associated with a pattern of breathing which can be recognized as abnormal, although some patients with gross disease have patterns of breathing indistinguishable from normal.

Continuous records of breathing may be useful in indicating a change in a patient's condition, as in developing carbon dioxide narcosis. Arterial puncture may be associated with a gross change in the pattern of breathing, and such recordings confirm the need for waiting until a stable state returns before withdrawing a blood sample.

\section{BREATH SOUNDS}

P. FORGACS The white noise of breathing heard at a distance with the unaided ear is often abnormally loud in patients with diffuse airways obstruction. Measurement of the mid-inspiratory breath sound, recorded through a microphone close to the mouth and correlated with instantaneous flow rate, confirms this clinical observation. The rise in the intensity of this sound for equal increments of flow rate is much greater in chronic bronchitis and asthma than in healthy subjects. Exercise, hyperventilation and cigarette smoke often intensify the breath sounds, while atropine and isoprenaline aerosol usually quietens them. The white noise of breathing is generated by turbulent flow in the mouth, larynx, and the first few generations of the airways. Variations in the loudness of the inspiratory sound reflect calibre changes in the large bronchi.

\section{THE LYMPHATICS OF THE THORAX}

\section{The Anatomy of the Lymphatics of the Lungs and Chest} Wall

DAVID H. TRAPNELL There are three main groups o pulmonary lymphatics in man, distinguished not only by their site but also by their origin. Some lymphatics begin at the periphery of the lung lobules, many of them thus lying in the pleura or interlobular septa. The pleural channels drain into the interlobular lymphatics, which join to give rise to the perivenous lymphatics.

A second group originates as blind-ended tubes in the centre of acini around the alveolar ducts. (There are no lymphatics in the walls of alveoli.) These vessels give rise to the peribronchial lymphatics which form a network around the broncho-arterial bundle to reach the hilum.

The third group of pulmonary lymphatics, lying in the interlobular septa of the depth of the lung, are anastomotic and join the peribronchial and perivenous groups together. 
Much less is known of the lymphatics of the pleura and chest wall. There are two main directions of lymph flow-forwards to the internal mammary chain of nodes and vessels, and backwards to the posterior mediastinum. Some of the lymphatics of the lower intercostal spaces drain downwards, through the diaphragm, to reach the cisterna chyli. Some of the mediastinal lymphatics communicate directly with the thoracic duct while the majority run upwards to join the paratracheal and pulmonary lymphatics and then drain by one or more vessels into the veins at the base of the neck near the thoracic duct.

The radiological appearances of the abnormal lymphatics in lymphangitis carcinomatosa, pulmonary oedema, etc., are discussed.

\section{Lymphography}

w. F. WHITE The lymphatics and lymph nodes draining many parts of the body may now be demonstrated by lymphography.

Visualization of the lymphatics is achieved by the subcutaneous injection of a suitable coloured material, and following cannulation radio-opaque material may be injected. In this way it is possible, by injecting the lymphatics on the dorsum of the foot, to visualize the inguinal, iliac and para-aortic nodes, together with the cisterna chyli, and the thoracic duct in some instances. The para-vertebral lymph nodes in the chest may be seen, but this is extremely variable.

The oily contrast medium enters the great veins and forms microemboli in the small pulmonary vessels. The complications of the technique are invariably manifest in the chest.

This communication concerns the demonstration of lymph nodes, the thoracic duct and the pulmonary complications of the technique.

\section{Chylothorax and Chylous Reflux}

K. ROsS A review is presented describing briefly the normal physiology of the thoracic duct and the aetiology of chylothorax.

The practical management of chylothorax is discussed in greater detail and the problem of chylous reflux, as it affects the pleural lymphatic system, is commented upon.

\section{Physiological and Pathological Mechanisms Governing Fluid Accumulation within the Pleural Space}

w. LECKIE Animal work illustrating the mechanisms responsible for preventing fluid accumulation within the pleural space is briefly reviewed. Predicted values for fluid and protein clearance in health are also deduced from animal studies.

Pathological mechanisms affecting fluid and protein balance within the pleural space are discussed, and the three factors (1) alterations in pleural capillary hydrostatic pressure, (2) alterations in pleural capillary permeability and (3) changes in lymphatic drainage, are related to different clinical conditions, e.g, pleural tuberculosis, malignancy, pulmonary infarct, congestive cardiac failure and Meigs's syndrome.

The effect of intrapleural corticosteroid administration on protein turnover in the pleural space is discussed.

Finally, in the light of these observations, a few $\stackrel{\mathbb{Q}}{\Omega}$ recommendations regarding terminology and manage- is ment are made.

\section{Clinical Manifestations of Disorders of the Pulmonary and Pleural Lymphatics}

PETER EMERSON The role of the lymphatics in pulmonary $\overrightarrow{\vec{x}}$ oedema is described with mention of drainage of the thoracic duct as a method of resolving intractable oedema in in congestive failure and cirrhosis of the liver. The iv concept of chylous reflux and its clinical presentation is of described together with the effect and the developmental ${ }_{2}$ abnormalities of the lymphatics of the lungs and pleura, $\mathrm{O}$ i.e., congenital pulmonary lymphangiectasis in infants and pulmonary and pleural disorders associated with $\bar{z}$ developmental lymphoedema in adults.

\section{SPONTANEOUS PNEUMOTHORAX IN CYSTIC FIBROSIS}

P. F. Mitchell-hesigs Five cases of cystic fibrosis complicated by eight pneumothoraces are described. These cases come from a group of 49 patients who have attended the Brompton Hospital between 1964 and 1969 for management of their cystic fibrosis. Three of the patients, who had a total of six pneumothoraces, were managed by pleurectomy. Spontaneous pneumothoraces were a terminal event in a fourth patient and an incidental finding which required no specific treatment in a fifth patient.

The possible aetiology of the pneumothorax in cystic fibrosis is discussed with particular consideration of the known alveolar hypoplasia and overdistension and the abnormality of the visceral pleura. The results of pleurectomy in three patients are discussed both in relation to the successful avoidance of further pneumothoraces and in the notable absence of a restrictive ventilatory defect.

In view of the relative success of pleurectomy it is suggested that this treatment should be considered for the management of any patient with cystic fibrosis who develops a spontaneous pneumothorax.

\section{PROBLEMS WITH LONG-TERM ENDOCARDIAL PACING IN CHILDREN}

A. H. M. SIDDONS, K. CHATTERJEE, A. M. HARRIS and $\omega$ M. PEARD Fortunately it is uncommon that a child needs long-term cardiac pacing. Such patients usually have 0 persistent complete heart block after cardiac surgery or $\stackrel{\odot}{\Phi}$ repeated Stokes Adams attacks associated with congenital $\stackrel{\oplus}{\oplus}$ (and sometimes acquired) complete heart block.

Eight children aged 7 months to 15 years have had long-term pacemakers implanted, and all are still being paced at 9 to 31 months. Complications encountered relate mainly to the need to lengthen the endocardial electrode with growth, and the implantation of a large foreign body. 